\title{
Research on Intelligent Driving Control System Based on Neural Network
}

\author{
Shengnan Liu ${ }^{1,2}$ \\ ${ }^{1}$ Shenyang institute of computing technology, Chinese academy of sciences, Shenyang 110000, China \\ ${ }^{2}$ school of Municipal Environmental Engineering, Jilin jianzhu University, Changchun 130000, China \\ 13756512317@163.com
}

Keywords: Operational stability; Neural network; Intelligent driving

\begin{abstract}
Based on the birth of the car, this paper provides a subjective survey with an emphasis on automotive technology. Intelligent driving will open the day of driving technology from now on. In recent years, a great deal of attention has been paid to intelligent control theory, and studies have been undertaken. The intelligent driving has gone to many family. This paper aims to deal with operational stability model and precision model of neural network based on the operating trend of automobile power system and analysis of forecast. This paper adopts optimization control algorithm to extend the service life of the car.
\end{abstract}

\section{Introduction}

Following the enterprise reform and scientific and technological progress. Automobile technology is moving from traditional machinery to intelligence. Cooperate with the demand of Industrial transformation, upgrade of living expenses. China has embraced technological progress with a series of new measures in its new scientific and technological transformation, improve the development speed of intelligent energy-saving technology[1].Our country is a big industrial country, It is also the world's second largest economy. The reform of automobile industry plays an important role in promoting the development of transportation. Hyundai motor control is usually based on a Can-line control. Therefore, there is a lot of development space in automobile optimization and deep learning algorithm.

China's professional aspects of computation and theoretical research on coupling of various components are still developing and updating, and the market is facing the consumer upgrading and people pay more and more attention to the high-quality lifestyle. In the automotive field, this provides a great development space and market opportunity for the development of driverless cars[2].

\section{Vehicle operation control structure}

The controller is designed to make targeted decision for the car such as vehicles and machines. The efforters are mechanical forces and moments on the mechanical system, such as the motor, the engine, launcher, the wheels[3]. Mechanical and electrical control systems are used to control the motion of the effector to the machine accordingly. The main parts of the intelligent vehicle power system are: Signal receiver, high - level control, low - level control, control distribution, actuator. By mechanical design, control structure design as shown (see Fig.1)

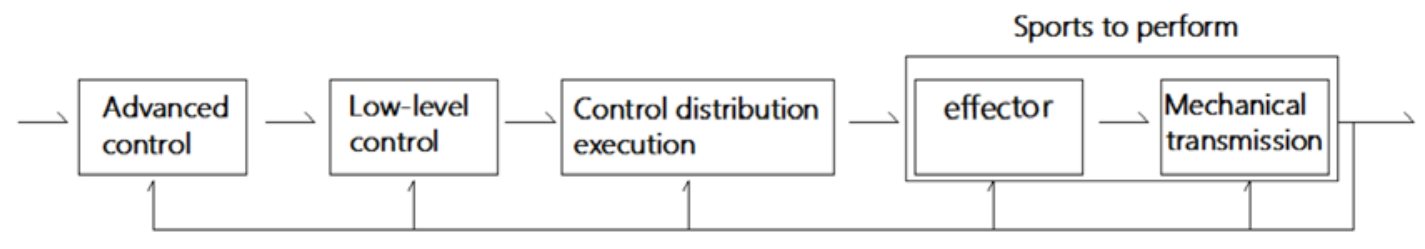

Fig. 1 structure diagram of automobile control system 
By mechanical design, a neural network control allocation algorithm is designed in order to improve the accuracy of internal control calculation, overall control is more coordinated. So we made a new algorithm is constructed for the precision and control time of the vehicle sensor. The over-actuated mechanical system, the controllability of the out put may be more stability and accuracy. It has effectively extended the service life of cars.

The single unit control of the control system is conducive to the upgrading and replacement of a single module of the system. In multi-task processing, it can balance the workload of all parts and improve the overall operation ability of the control system. As neural network control algorithms become more and more common, downstream controls and effectors can be developed to upstream central controllers. Think of it as a car control effect similar to human local conditioning. Reduce in emergencies, slow vehicle control operations, and improve the sensitivity of the motion effectors.

\section{Stable operation and neural algorithm modeling}

\subsection{Travel stability model}

Travel stability can achieve a directive transient response effects for driving condition of thecar. The sensor is based on the frequency is consistent to collect the number, minimization are handled system operation workload and provide convenient running conditions for the internal operations.

In the experiments, be make sure the initial data to be more accurate, the center point of the car is the data sampling point. Avoid the tire bump with the ground ineffective signal interference. The distance traveled by the car at the adjacent signal point is“"s".The speed is controlled by the driver.

(1) The moving mean of steady operation:

$$
\mathrm{S}_{\mathrm{t}}=\mathrm{Y}_{\mathrm{t}}
$$

(2) Based on the(1),for $t+1$ moment, the resulting moving average, The resulting moving average:

$$
\mathrm{S}_{\mathrm{t}+1}=\beta \mathrm{Y}_{\mathrm{t}}+\beta(1-\beta) \mathrm{Y}_{\mathrm{t}}+\beta(1-\beta)^{2} \mathrm{Y}_{\mathrm{t}}+\ldots
$$

$\mathrm{S}_{\mathrm{t}}$ is the actual distance traveled per unit time; $\mathrm{Y}_{\mathrm{t}}$ is the speed of the car at this point, $\mathrm{m} / \mathrm{s} ; \beta$ is the linear regression coefficient, according to road conditions between 0 -1.Stable running performance is $/ S_{t} \div S_{t+1} / \leq 0.01$, the better ideal.

\subsection{Precision model of neural network}

The neural network algorithm is based on the structure of human neurons, The neural network algorithm is generated by this structure. Different algorithms adapt to different situations, Therefore, the specific neural network structure is used to predict the running time and running state. This paper proposed a derivative variable is data training, optimization, output. In view of the diversity of the algorithm, we choose to analyze the evaluation algorithm for vehicle operation control. It considers a class of parameter uncertainty which is sensor sampling time delay and the system calculation algorithm is not accurate.

The detection index and the basis of neural network data operation. Such as formula:

$$
\begin{gathered}
\mathrm{NE}=(1 / \mathrm{Y})^{*}\left(\Sigma / \mathrm{e}_{\mathrm{t}} /\right) \\
\mathrm{MAE}=(\text { Validation data set } \mathrm{NE}) /(\text { The training set } \mathrm{NE})=\mathrm{NE} /\left\{[1 /(\mathrm{n}-1)]^{*} \Sigma / \mathrm{Y}_{\mathrm{t}+1}-\mathrm{Yt} /\right\}
\end{gathered}
$$

The neural network algorithm is based on the human neuron structure formula: NE is the absolute error of this trip; $Y$ is the average driving speed of this time; $e_{t}$ is the error of predicting operation stability (the absolute value of predicting speed and actual speed); $n$ is the number of experiments. Neural network precision model for vehicle running speed, the conclusion is shown(see Fig.2): 


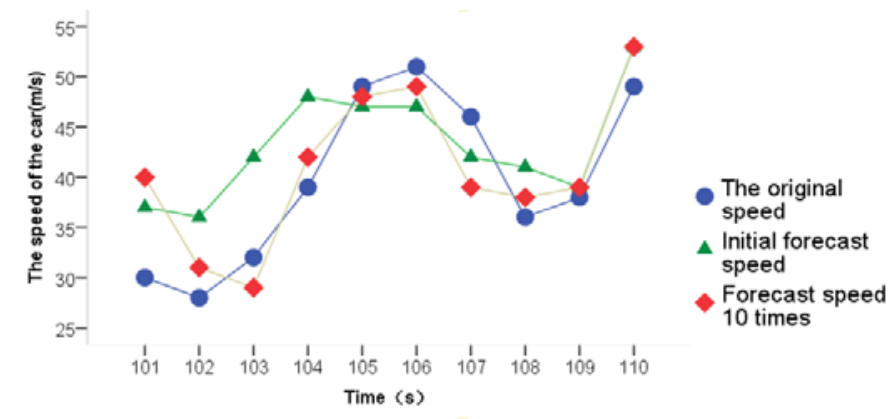

Fig 2. Actual speed and predicted speed

The picture showd us in the comparison between the actual speed and the prediction model.In process control trend of the speed prediction following the number of operations increases tend to be more obvious. Based on neural network analysis achieve a good transient on the speed accuracy model is used to predict the change trend of speed.

By increasing the prediction calculation times, we find that the model of the modified neural network tends to be stable and the curve features are more obvious. As a result, the calculated results tend to be ideal when the data volume and calculation volume increase. The model is also applicable to algorithms of computer self-learning and big data analysis.

\section{Conclusion}

By the advantage of the neural network algorithmmodel, prediction of vehicle running stability and speed trend. We conclude that the study of current speed prediction mainly focuses on three aspects:

(1) In process control of central controller, the speed stability can determined the comfort and safety of cabin crew. Any date of out put by central controller are commonly exploited via model and real-time optimization, which are time-variable optimization-based control.

(2) A controller is designed to achieve not only optimize the vehicle's variable speed air time, but also improve engine drive sensitivity. More accurate analysis with some perspectives on new applications and theoretical in intelligent driving.

(3) This paper has proposed a method for analysis of neural network speed control, resolve with forecast param-eter uncertainties independently. The concepts, experiments data processing and other experimental content and methods are introduced. Refer to other fields.

\section{References}

[1] Natasha Merat, ${ }^{\mathrm{a},}$,Tyron Louw $^{\mathrm{a}}$,Ruth Madigan $^{\mathrm{a}}$, Marc Wilbrink ${ }^{\mathrm{b}}$, Anna Schieben ${ }^{\mathrm{b}}$. What externally presented information do VRUs require when interacting with fully Automated Road Transport Systems in shared space[J]. Accident Analysis and Prevention,2018,03(018):1-9.

[2] Amitai Etzioni, Oren Etzioni. AI assisted ethics[J]. Ethics 1nf Technol, 2016,18:149-156

[3] M. Hirata*, K.Z. Liu* and T. Mita**.ACTIVE VIBRATION CONTROL OF A 2-MASS SYSTEM USING $\mu$-SYNTHESIS WITH A DESCRIPTOR FORM REPRESENTATION.[J] Pergamon,1996, 4(4):545-552. 